
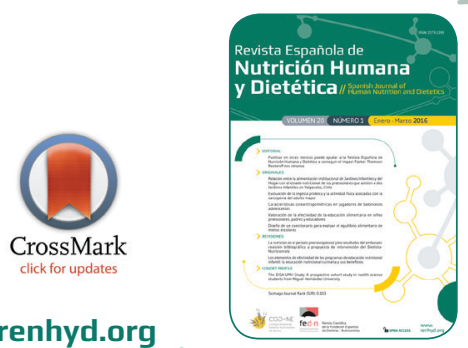

www.renhyd.org

ORIGINAL

\title{
Características cineantropométricas en jugadores de baloncesto adolescentes
}

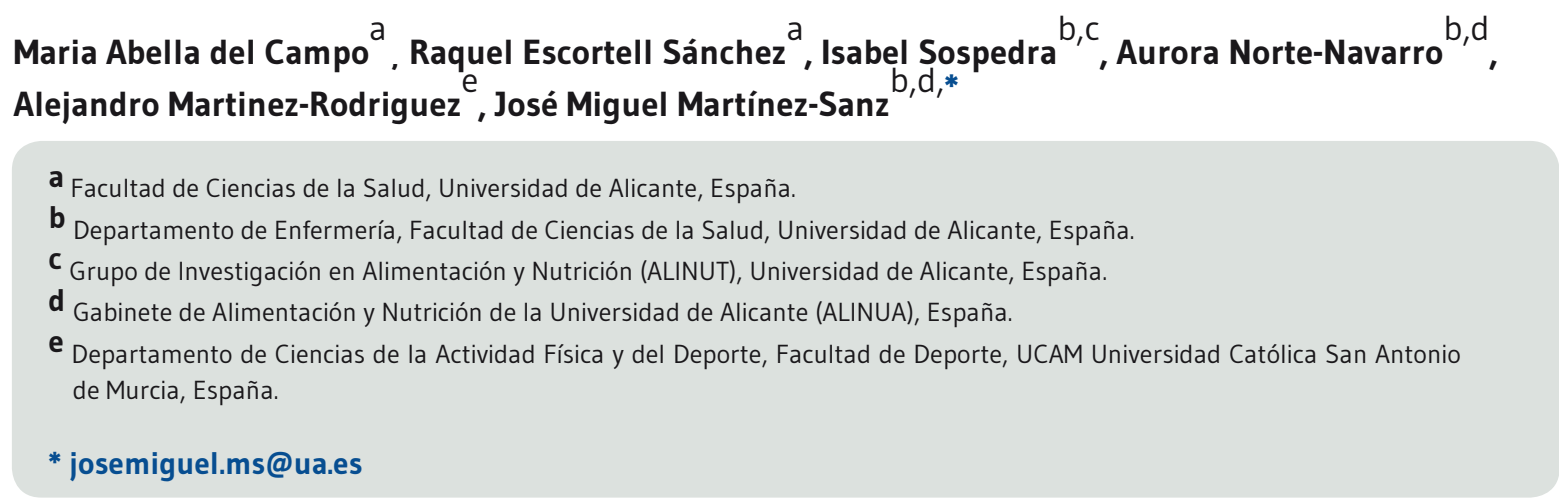

Recibido el 21 de agosto de 2015; aceptado el 9 de diciembre de 2015.

Características cineantropométricas en jugadores de baloncesto adolescentes

\section{PALABRAS CLAVE}

Antropometría;

Composición corporal;

Somatotipo;

Baloncesto.

\section{RESUMEN}

Introducción: El baloncesto presenta una gran heterogeneidad antropométrica entre jugadores en relación a la posición de juego. El base suele ser el más bajo del equipo pero también el más rápido. El escolta suele ser similar al base, más bajo, rápido y ágil que el resto. El alero es de una altura intermedia entre los jugadores interiores y exteriores, combina la altura con la velocidad, y el pívot tiene un rol más físico, es el más alto y con mayor masa muscular. El objetivo del estudio es describir el perfil cineantropométrico del equipo cadete masculino del Valencia Basket Club.

Material y Métodos: Se han evaluado 20 jugadores de baloncesto cadete masculino (15,35£0,59años). Un antropometrista nivel I tomó las medidas según la metodología de la Society for the Advancement of Kinanthropometry (ISAK). Se calculó la composición corporal según el consenso de cineantropometría del Grupo Español de Cineantropometría (GREC), el somatotipo según Heath-Carter, e índices adiposo-musculares.

Resultados: Se observaron diferencias en los valores de peso, talla, pliegues cutáneos y composición corporal, de las variables estudiadas de la muestra total y por posición de juego, siendo los valores menores los de la posición base y mayores en los pívots. Se estableció una tendencia creciente de estos parámetros en el siguiente orden: base, escolta, alero y pívot.

Conclusiones: El somatotipo global del jugador de baloncesto cadete del Valencia Basket Club es ectomorfo balanceado. Conocer el perfil cineantropométrico puede beneficiar la elaboración de planes de entrenamiento y nutrición, así como reflejar la evolución y dirigirla hacia las características ligadas al rendimiento óptimo. 
Kinanthropometric characteristics in teenager basketball players

\section{KEYWORDS}

Anthropometry;

Body Composition;

Somatotypes;

Basketball.

\section{ABSTRACT}

Introduction: Basketball is a sport with a high heterogeneity anthropometric as playing position. Point guard is usually the lowest player but also the fastest. Guard is similar to point guard, faster and more agile than the others. Small forward has an intermediate height between the inside and outside players and it combines height with velocity. And center (pivot) has a physical role, it has the highest and more muscle mass. The aim of this study is to describe the kinanthropometric profile of male cadet "Valencia Basket Club".

Material and Methods: A total of 20 male cadet basketball players were evaluated $(15.35 \pm 0.59$ years old). An anthropometrist level 1 took anthropometric measures according to the methodology of the International Society for the Advancement of Kinanthropometry (ISAK). Body composition was calculated according to the kinanthropometry consensus of the Kinanthropometry Spanish Group (GREC), somatotype with Heath-Carter's method and adiposemuscular and cardiovascular risk index.

Results: Differences in values of weight, height, skinfold and body composition from variables of sample and position were observed. Lower values were from point guard position and higher values were from center position. An increasing trend of these parameters was established in the following order: point guard, guard, small forward and center.

Conclusions: The Valencia Basket Club cadet player overall somatotype is balance ectomorph. To know kinanthropometric profile may be beneficial to develop specific training and nutrition plans as well as to see evolution and characteristics for optimal performance.

\section{CITA}

Abella del Campo M, Escortell Sánchez R, Sospedra I, Norte-Navarro A, Martinez-Rodriguez A, Martínez-Sanz JM. Características cineantropométricas en jugadores de baloncesto adolescentes. Rev Esp Nutr Hum Diet. 2016; 20(1): 23 - 31. DOI: 10.14306/renhyd.20.1.179

\section{INTRODUCCIÓN}

El baloncesto es un deporte de contacto físico, en el cual se enfrentan 2 equipos de 5 jugadores cada uno, con un máximo de 12 jugadores intercambiables ${ }^{1}$. Estos se agrupan según la posición de juego diferenciándose entre: a) Base: dirige el juego en la ofensiva. Su función se basa en hacer llegar el balón a sus compañeros en la mejor posición posible para poder anotar. Suele ser el que mejor maneja y pasa el balón de su equipo. En muchas ocasiones, suele ser el jugador más bajo y más rápido del equipo; b) Escolta: tiene la responsabilidad de anotar desde el perímetro y convertir puntos para su equipo. Generalmente es más alto que el base y suele realizar una mayor cantidad de lanzamientos; c) Alero: tiene la responsabilidad de ser un sobresaliente anotador y buen defensor. La versatilidad es casi siempre una de las grandes características de un buen alero ya que tiene que ser grande para jugar cerca de la canasta pero también lo suficientemente rápido para anotar desde el perímetro; d) Alapívot: generalmente destaca en los elementos más físicos del juego como son los rebotes y la defensa. Es casi siempre el jugador más fuerte del equipo. Pasa la mayor cantidad del tiempo en el área cercana a la canasta; e) Pívot: muy importante ya que el equipo depende de él en ambos tableros. En la capacidad ofensiva, debe tener la capacidad de anotar cerca de la canasta mientras que, en la defensa, tiene la responsabilidad de coger los rebotes y bloquear lanzamientos. Suele ser el jugador más alto del equipo². 
La cineantropometría es el área de estudio de las ciencias del deporte que relacionan las medidas corporales en su forma, proporciones y composiciones con la función ${ }^{3}$, la cual se considera un área básica dentro de la valoración médica del deportista. Fue presentada en 1976 por William Ross como el estudio del tamaño, forma, proporcionalidad, composición, maduración biológica y función corporal, con el objeto de comprender el proceso de crecimiento, el ejercicio, el rendimiento deportivo y la nutrición. Desde los años 80, se valora el estudio cineantropométrico de los deportistas como una herramienta más para mejorar el rendimiento deportivo de éstos ${ }^{4}$. La técnica antropométrica permite medir el peso corporal, la estatura, envergadura y diferentes pliegues cutáneos, perímetros, diámetros y longitudes. Mediante los datos obtenidos, y gracias a la aplicación de diferentes fórmulas, se puede obtener información sobre el somatotipo, la composición corporal y la proporcionalidad de diferentes partes del cuerpo 5 .

El somatotipo es usado para describir el tipo físico más susceptible a determinadas enfermedades y como medio de relacionar el tipo corporal con éxito en varias modalidades deportivas $^{6}$. Se cualifican las constituciones físicas del hombre en función de tres componentes, la cantidad de grasa corporal, la masa muscular y el tejido óseo, en sus debidas proporcionalidades, consiguiendo una forma fotográfica del perfil del deportista. El somatotipo de Heath-Carter ${ }^{7}$ ha sido definido como una descripción cuantitativa de la conformación morfológica actual o presente del cuerpo.

De cada uno de los elementos de la composición corporal no existe un valor determinante que por sí solo determine el éxito deportivo. En cambio, en los jugadores de baloncesto se cumplen unas tendencias donde el base posee menor estatura y menor peso al igual que menor porcentaje de masa grasa y masa muscular, aumentando estos 4 valores hasta llegar al pívot, el cual es más alto y también más pesado, además de disponer de mayor masa grasa y muscular ${ }^{8,9}$.

En cuanto al somatotipo predominante en deportes de equipo como el baloncesto, son mesoectomorfos, ectomesomorfos, endomesomorfos y mesomorfos balanceados ${ }^{10}$.

El objetivo del estudio es describir el perfil cineantropométrico de los cadetes masculino del Valencia Basket Club según posición de juego.

\section{MATERIAL Y MÉTODOS}

Estudio observacional y descriptivo de las características cineantropométricas en jugadores de baloncesto adolescentes.

\section{Muestra}

20 jugadores masculinos de baloncesto de la categoría Cadete $A$ y $B$ del Valencia Basket Club.

\section{Instrumentos y Procedimiento}

Para la valoración cineantropométrica se siguieron las normas y técnicas de medición recomendadas por la International Society for the Advancement of Kinanthropometry $\left(\right.$ ISAK) ${ }^{11}$ y el Grupo Español de Cineantropometría (GREC) 4 . Antes de comenzar la toma de medidas cineantropométricas se informó a los sujetos sobre la finalidad del estudio. Se obtuvo un consentimiento informado firmado por los responsables del estudio, así como por el médico del Valencia Basket Club. Cada participante y sus respectivos padres o tutores legales también lo firmaron. Todo ello, al amparo de las directrices éticas dictadas en la declaración de Helsinki de la Asociación Médica Mundial, para la investigación con seres humanos.

Se utilizó el siguiente material antropométrico: a) tallímetro de pared (precisión 1mm); b) báscula (precisión 100g); c) cinta métrica Cescorf (precisión $1 \mathrm{~mm}$ ); d) paquímetro Cescorf (precisión 1mm); e) plicómetro Cescorf (precisión $1 \mathrm{~mm})$; y f) material complementario (lápiz dermográfico negro y blanco para marcar al sujeto; y banco antropométrico (medidas 40×50×30)).

Un medidor acreditado ISAK de nivel I tomó las siguientes medidas necesarias para la valoración antropométrica, teniendo en cuenta el error técnico de medición intraobservador indicado por la ISAK (2011) 12 $^{2}$ para nivel I (el 10,0\% para pliegues y el 2,0\% para perímetros y diámetros): a) medidas básicas (peso, talla y envergadura); b) pliegues cutáneos (tríceps, subescapular, bíceps, ileocrestal, supraespinal, abdominal, muslo anterior y pierna medial); c) perímetros (brazo relajado, brazo contraído y flexionado, cintura, cadera, muslo medio y pierna); y d) diámetros (húmero, muñeca y fémur). Mediante las fórmulas descritas en el consenso de cineantropometría del $\mathrm{GREC}^{13}$, se calculó la composición corporal mediante el modelo de tres componentes: a) masa grasa mediante la ecuación de Withers ${ }^{14}$; b) masa muscular mediante la propuesta de Lee ${ }^{15}$; y c) masa ósea mediante la de Rocha ${ }^{16}$. También se calculó el sumatorio de 8 pliegues (tríceps, bíceps, subescapular, ileocrestal, supraespinal, abdominal, muslo anterior y pierna medial), el índice de masa corporal (IMC), el índice cintura-cadera (ICC), el índice músculo-óseo (IMO) y el índice de distribución de la grasa (IDG).

Para el cálculo del somatotipo se determinó el somatotipo medio, los tres componentes del somatotipo por separado (endomorfia, mesomorfia, ectomorfia), siguiendo el método de Heath-Carter ${ }^{7,17}$. Todos los parámetros se determinaron y clasificaron según posición de juego. 


\section{Análisis estadístico}

Los datos recopilados fueron analizados mediante la versión 22.0 del software IBM SPSS Statistics ${ }^{\circledR}$ para Windows. Se efectuaron análisis de estadísticos descriptivos, cuyos resultados se expresaron como media \pm desviación estándar de la media. La normalidad de las variables de estudio se comprobó mediante el test Kolmogorov-Smirnov. Las comparaciones de medias, se llevaron a cabo a través de la prueba ANOVA, para analizar las posibles diferencias en función de las distintas posiciones, así como las correspondientes pruebas post-hoc (Tukey y Games-Howell) en función de si se cumplía o no homogeneidad de las varianzas. Se consideró la existencia de una diferencia estadísticamente negativa cuando el valor de $p$ era menor o igual a 0,05.

\section{RESULTADOS}

Los resultados obtenidos sobre las características cineantropométricas de la composición corporal se muestran en la Tabla 1 según el total de la muestra y por posición de juego. En general, se observó un aumento de los valores de peso, talla, pliegues cutáneos y composición corporal según la posición de juego, siendo los valores menores los de la posición base y mayores en los pívot. Se estableció una tendencia creciente de estos parámetros en el siguiente orden: base, escolta, alero y pívot. De las características cineantropométricas de la muestra estudiada destacaron:

- Medidas básicas: el peso medio del total de la mues-

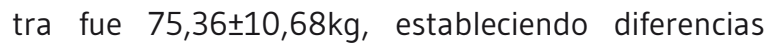
entre los jugadores base $(65,95 \pm 11,31 \mathrm{~kg})$ y pívot $(85,36 \pm 10,23 \mathrm{~kg})$. Respecto a la talla, se observó un crecimiento del valor de la misma, según la posición de juego con diferencias estadísticamente significativas de la posición base $(173,65 \pm 6,63 \mathrm{~cm})$ con escolta $(185,00 \pm 6,36 \mathrm{~cm})$, alero $(186,96 \pm 4,19 \mathrm{~cm})$ y pívot $(193,60 \pm 2,23 \mathrm{~cm})$. La envergadura también mostró una tendencia creciente como el peso y la talla, pero hayamos diferencias significativas entre la posición base $(180,9 \pm 4,64 \mathrm{~cm})$, con alero $(192,70 \pm 7,42 \mathrm{~cm})$ y pívot $(197,40 \pm 4,16 \mathrm{~cm})$.

- Pliegues cutáneos: el valor medio del sumatorio de 8 pliegues de la muestra fue $87,27 \pm 22,62 \mathrm{~mm}$, siendo el mayor valor en los pívot $(105,14 \pm 30,40 \mathrm{~mm})$ y más bajo en los base $(75,68 \pm 18,74 \mathrm{~mm})$. En los valores de pliegues cutáneos y sumatorio, también se observó aumento según la posición de juego, pero sin diferencias estadísticamente significativas.
- Perímetros y diámetros: se vio ausencia de riesgo cardiovascular, además de diferencia significativa entre el perímetro de la cadera de los jugadores base $(93,18 \pm 5,91 \mathrm{~cm})$ y pívot $(102,66 \pm 5,63 \mathrm{~cm})$, y diámetro de la muñeca de la posición alero $(5,71 \pm 0,18 \mathrm{~cm})$ y pí$\operatorname{vot}(6,10 \pm 0,14 \mathrm{~cm})$.

- Composición corporal: se observó un aumento de los valores de masa grasa, muscular y ósea según la posición de juego. El IDG fue en todos los casos $>1$, es decir, que la cantidad de grasa relativa de las extremidades fue mayor que la del tronco. Por último, el IMO (relación entre kilos de músculo y kilos de hueso) fue bajo, inferior a 3,4.

- Somatotipo: en general fue ectomorfo balanceado y, concretamente, los jugadores base mesoectomorfos, escoltas y aleros ectomorfos balanceados, y pívots ectoendomorfos. El valor de endomorfia del grupo

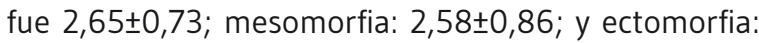
$3,71 \pm 1,14$. Es decir, jugadores altos, con poca grasa subcutánea y bajo desarrollo muscular, pero con contornos musculares y óseos visibles.

La Figura 1 muestra la distribución del somatotipo de los jugadores de baloncesto cadete masculino según la posición de juego, así como el somatotipo general.

\section{DISCUSIÓN}

En general, el peso corporal de los jugadores de baloncesto cadete masculino está entre 63,48 y $94,4 \mathrm{~kg}^{18,19}$. En nuestro estudio, los cadetes se sitúan en un valor intermedio $(75,36 \pm 19,68 \mathrm{~kg})$.

La altura del jugador de baloncesto cobra especial importancia y la talla media del jugador cadete masculino va de 173,20 a $198,4 \mathrm{~cm}^{18,19}$, siendo también un valor intermedio el hallado en nuestro estudio $(185,76 \pm 8,08 \mathrm{~cm})$.

En cuanto al IMC, según la clasificación de la OMS, el valor medio del estudio $\left(21,78 \pm 2,26 \mathrm{~kg} / \mathrm{m}^{2}\right)$ está dentro de la normalidad. No obstante, hay que tener en cuenta que el IMC describe la cantidad de masa y no la calidad de ésta, por lo que no es considerado un método adecuado para estimar el sobrepeso cuando los deportistas no han alcanzado el desarrollo muscular óptimo que corresponde a sus requerimientos de disciplina en particular, puesto que cambios en el porcentaje muscular tendrán su influencia sobre el IMC20. Este es un factor a tener presente en este estudio, ya que la muestra es de 15,35£0,59 años, en fase de crecimiento y desarrollo osteomuscular. 
Rev Esp Nutr Hum Diet. 2016; 20(1): 23 - 31

Características cineantropométricas en jugadores de baloncesto adolescentes

Tabla 1. Características cineantropométricas de la muestra.

\begin{tabular}{|c|c|c|c|c|c|}
\hline & Total $(n=20)$ & Base $(n=4)$ & Escolta $(n=2)$ & Alero $(n=9)$ & Pívot $(n=5)$ \\
\hline \multicolumn{6}{|l|}{ 1. MEDIDAS BÁSICAS } \\
\hline Edad $( \pm D E)$ & $15,35( \pm 0,59)$ & $15,25( \pm 0,96)$ & $15,50( \pm 0,71)$ & $15,33( \pm 0,50)$ & $15,40( \pm 0,55)$ \\
\hline Peso (kg) ( $\pm \mathrm{DE})$ & $75,36( \pm 10,68)$ & $65,95( \pm 11,31)^{p *}$ & $76,05( \pm 5,59)$ & $73,83( \pm 7,57)$ & $85,36( \pm 10,23)^{B *}$ \\
\hline Talla $(\mathrm{cm})( \pm \mathrm{DE})$ & $185,76( \pm 8,08)$ & $173,65( \pm 6,63)$ E* A** P*** & $185,00( \pm 6,36)^{B} *$ & $186,96( \pm 4,19)^{\mathrm{B} * *}$ & $193,60( \pm 2,23)^{B * * *}$ \\
\hline Envergadura $(\mathrm{cm})( \pm \mathrm{DE})$ & $191,78( \pm 8,08)$ & $180,95( \pm 4,64)^{\text {A* P* }}$ & $195,25( \pm 2,47)$ & $192,70( \pm 7,42)^{\mathrm{B} *}$ & $197,40( \pm 4,16)^{B *}$ \\
\hline $\mathrm{IMC}(\mathrm{kg} / \mathrm{m} 2)( \pm \mathrm{DE})$ & $21,78( \pm 2,26)$ & $21,74( \pm 2,43)$ & $22,20( \pm 0,10)$ & $21,15( \pm 2,36)$ & $22,76( \pm 2,55)$ \\
\hline \multicolumn{6}{|l|}{ 2. PLIEGUES CUTÁNEOS (mm) } \\
\hline Tríceps ( \pm DE) & $10,71( \pm 2,98)$ & $9,33( \pm 3,36)$ & $10,25( \pm 1,06)$ & $9,92( \pm 2,65)$ & $13,42( \pm 2,63)$ \\
\hline Subescapular ( \pm DE) & $9,36( \pm 2,13)$ & $9,13( \pm 1,80)$ & $9,25( \pm 0,35)$ & $8,79( \pm 1,30)$ & $10,60( \pm 3,60)$ \\
\hline Bíceps ( \pm DE) & $5,23( \pm 1,15)$ & $4,50( \pm 0,41)$ & $5,00( \pm 0,00)$ & $5,17( \pm 1,20)$ & $6,00( \pm 1,41)$ \\
\hline lleocrestal ( $\pm \mathrm{DE})$ & $15,08( \pm 5,61)$ & $12,95( \pm 3,30)$ & $12,15( \pm 1,63)$ & $14,16( \pm 5,31)$ & $19,62( \pm 6,92)$ \\
\hline Supraespinal ( $\pm D E)$ & $8,95( \pm 3,43)$ & $8,00( \pm 3,34)$ & $6,50( \pm 0,71)$ & $8,96( \pm 2,33)$ & $10,66( \pm 5,37)$ \\
\hline Abdominal ( \pm DE) & $13,60( \pm 5,20)$ & $11,83( \pm 3,98)$ & $12,00( \pm 2,83)$ & $13,12( \pm 4,46)$ & $16,52( \pm 7,68)$ \\
\hline Muslo ( \pm DE) & $13,96( \pm 2,72)$ & $11,33( \pm 2,47)$ & $13,90( \pm 1,27)$ & $14,32( \pm 2,78)$ & $15,42( \pm 2,20)$ \\
\hline Pierna ( $\pm \mathrm{DE})$ & $10,40( \pm 2,97)$ & $8,63( \pm 1,89)$ & $9,40( \pm 1,27)$ & $10,01( \pm 2,09)$ & $12,90( \pm 4,29)$ \\
\hline Sumatorio 8 pliegues $( \pm D E)$ & $87,27( \pm 22,62)$ & $75,68( \pm 18,74)$ & $78,45( \pm 2,05)$ & $84,44( \pm 18,06)$ & $105,14( \pm 30,40)$ \\
\hline \multicolumn{6}{|l|}{ 3. PERÍMETROS (cm) } \\
\hline Brazo relajado ( $\pm \mathrm{DE})$ & $28,49( \pm 2,55)$ & $26,90( \pm 2,63)$ & $28,65( \pm 1,77)$ & $28,26( \pm 2,54)$ & $30,12( \pm 2,47)$ \\
\hline Brazo flexionado y contraído ( $\pm D E)$ & $30,07( \pm 2,48)$ & $29,45( \pm 3,16)$ & $30,05( \pm 3,46)$ & $29,38( \pm 2,33)$ & $31,46( \pm 2,15)$ \\
\hline Cintura (mín.) ( $\pm D E)$ & $77,33( \pm 4,55)$ & $75,48( \pm 2,53)$ & $78,75( \pm 1,77)$ & $76,43( \pm 4,17)$ & $79,86( \pm 6,61)$ \\
\hline Cadera (máx.) ( \pm DE) & $97,57( \pm 5,46)$ & $93,18( \pm 5,91)^{\mathrm{P} *}$ & $99,25( \pm 4,03)$ & $96,32( \pm 3,40)$ & $102,66( \pm 5,63)^{B *}$ \\
\hline ICC ( $\pm \mathrm{DE})$ & $0,79( \pm 0,03)$ & $0,81( \pm 0,03)$ & $0,79( \pm 0,01)$ & $0,79( \pm 0,02)$ & $0,78( \pm 0,03)$ \\
\hline Muslo ( \pm DE) & $51,27( \pm 4,31)$ & $49,95( \pm 4,76)$ & $49,90( \pm 3,39)$ & $50,83( \pm 4,41)$ & $53,66( \pm 4,42)$ \\
\hline Pierna ( $\pm D E)$ & $37,05( \pm 2,38)$ & $35,35( \pm 2,41)$ & $36,80( \pm 1,27)$ & $36,69( \pm 2,26)$ & $39,14( \pm 1,80)$ \\
\hline \multicolumn{6}{|l|}{ 4. DIÁMETROS (cm) } \\
\hline Húmero ( $\pm \mathrm{DE})$ & $7,04( \pm 0,43)$ & $6,73( \pm 0,56)$ & $6,95( \pm 0,64)$ & $7,08( \pm 0,43)$ & $7,26( \pm 0,15)$ \\
\hline Muñeca ( \pm DE) & $5,79( \pm 0,33)$ & $5,60( \pm 0,48)$ & $5,70( \pm 0,57)$ & $5,71( \pm 0,18)^{\mathrm{P} * * *}$ & $6,10( \pm 0,14)^{A * *}$ \\
\hline Fémur ( $\pm \mathrm{DE})$ & $9,75( \pm 0,33)$ & $9,65( \pm 0,52)$ & $9,50( \pm 0,14)$ & $9,73( \pm 0,28)$ & $9,94( \pm 0,28)$ \\
\hline \multicolumn{6}{|l|}{ 5. COMPOSICIÓN CORPORAL } \\
\hline \% Graso Withers ( \pm DE) & $12,67( \pm 3,04)$ & $11,03( \pm 2,66)$ & $11,63( \pm 0,07)$ & $12,33( \pm 2,34)$ & $15,00( \pm 4,17)$ \\
\hline Peso Graso (kg) & $9,78( \pm 3,55)$ & $7,47( \pm 2,85)$ & $8,84( \pm 0,59)$ & $9,15( \pm 2,12)$ & $13,12( \pm 4,90)$ \\
\hline \% Muscular Lee ( \pm DE) & $41,75( \pm 2,26)$ & $43,59( \pm 3,19)$ & $40,96( \pm 0,03)$ & $42,21( \pm 0,98)$ & $39,79( \pm 2,40)$ \\
\hline Peso Muscular (kg) & $31,29( \pm 3,39)$ & $28,48( \pm 3,14)$ & $31,15( \pm 2,26)$ & $31,18( \pm 3,51)$ & $33,78( \pm 2,37)$ \\
\hline \% Óseo Rocha ( \pm DE) & $17,46( \pm 1,61)$ & $17,56( \pm 0,89)$ & $16,52( \pm 0,59)$ & $17,76( \pm 1,82)$ & $17,22( \pm 2,08)$ \\
\hline Peso Óseo (kg) & $13,05( \pm 1,36)$ & $11,53( \pm 1,65)$ & $12,58( \pm 1,37)$ & $13,01( \pm 0,68)$ & $15,54( \pm 0,38)$ \\
\hline \% Residual ( \pm DE) & $28,11( \pm 1,89)$ & $27,82( \pm 1,82)$ & $30,88( \pm 0,48)$ & $27,70( \pm 1,73)$ & $27,99( \pm 2,03)$ \\
\hline Peso Residual (kg) & $21,25( \pm 3,65)$ & $18,47( \pm 4,03)$ & $23,47( \pm 1,36)$ & $20,50( \pm 2,97)$ & $23,92( \pm 3,56)$ \\
\hline \multicolumn{6}{|l|}{ 6. ÍNDICES CORPORALES } \\
\hline Í. Músculo-Óseo (IMO) & $2,41( \pm 0,23)$ & $2,48( \pm 0,14)$ & $2,48( \pm 0,09)$ & $2,40( \pm 0,31)$ & $2,32( \pm 0,14)$ \\
\hline Í. Distribución Grasa (IDG) & $1,14( \pm 0,21)$ & $1,02( \pm 0,08)$ & $1,22( \pm 0,27)$ & $1,14( \pm 0,20)$ & $1,20( \pm 0,29)$ \\
\hline \multicolumn{6}{|l|}{ 7. SOMATOTIPO } \\
\hline Endomorfia ( $\pm \mathrm{DE}$ ) & $2,65( \pm 0,73)$ & $2,57( \pm 0,74)$ & $2,38( \pm 0,17)$ & $2,52( \pm 0,65)$ & $3,07( \pm 0,99)$ \\
\hline Mesomorfia ( \pm DE) & $2,58( \pm 0,86)$ & $3,38( \pm 0,64)$ & $2,49( \pm 0,16)$ & $2,40( \pm 0,97)$ & $2,31( \pm 0,70)$ \\
\hline Ectomorfia ( $\pm \mathrm{DE})$ & $3,71( \pm 1,14)$ & $3,00( \pm 0,92)$ & $3,39( \pm 0,32)$ & $4,10( \pm 1,24)$ & $3,69( \pm 1,23)$ \\
\hline
\end{tabular}

* La diferencia de medias es significativa al nivel 0,05. * * La diferencia de medias es significativa al nivel 0,01. $\mathrm{B}=$ Base; $\mathrm{A}=$ Alero; $\mathrm{E}=$ Escolta; $\mathrm{P}=$ Pívot. Las letras iniciales indican que el grupo en cuestión tiene diferencias significativas con respecto al grupo con la letra en superíndice. 
Figura 1. Somatocarta jugadores baloncesto cadete masculino.

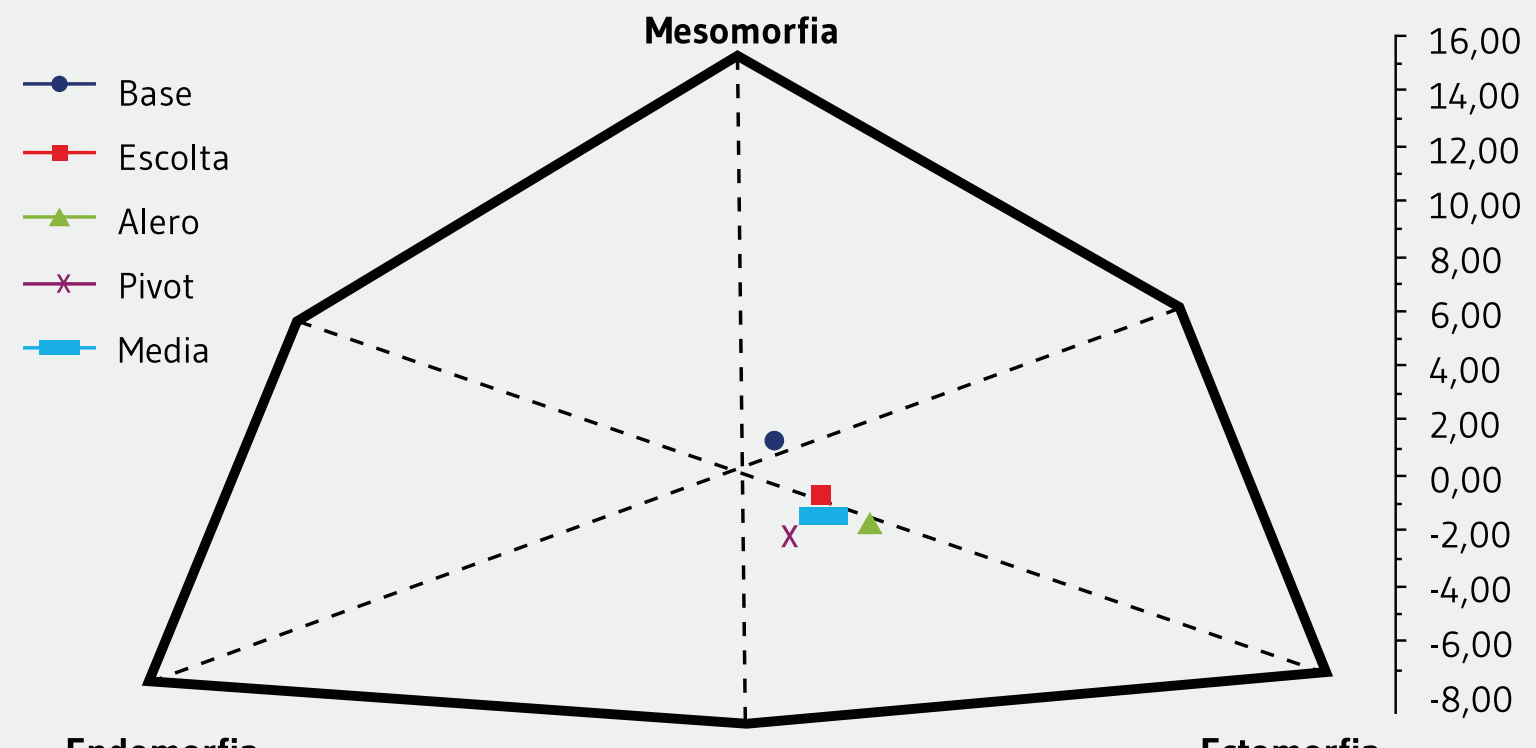

Endomorfia

Ectomorfia

$-8,00-7,00-6,00-5,00-4,00-3,00-2,00-1,000,00$ 1,00 2,00 3,00 4,00 5,00 6,00 7,00 8,00

Para calcular el porcentaje de grasa corporal a partir de diferentes variables antropométricas, la ecuación de Faulkner ${ }^{21}$ utiliza 4 pliegues correspondientes al tronco superior y, en cambio, la ecuación de Withers, $1987^{14}$ utiliza 7 pliegues correspondientes al tronco superior e inferior. La selección de la ecuación a utilizar para determinar el porcentaje de grasa corporal es una de las principales causas de error. Normalmente se utiliza el sumatorio de 6 pliegues, correspondiente a tríceps, subescapular, supraespinal, abdominal, muslo anterior y pierna medial, aunque también se utiliza el sumatorio de 8 pliegues, además de poder hacer una distinción entre la extremidad superior (tríceps, bíceps), inferior (muslo anterior, pierna medial) y tronco (subescapular, ileocrestal, supraespinal, abdominal) ${ }^{22}$.

Encontramos valores de $12,88 \%$ y $14,45 \%$ de masa grasa corporal $^{18}$ utilizando la fórmula de Faulkner ${ }^{21}$, siendo en nuestro estudio de $12,67 \%$ mediante la fórmula de Withers, $1987^{14}$. Datos similares se han observado en un estudio en 86 jugadores de baloncesto ${ }^{23}$. Así pues, se puede decir que el porcentaje graso de nuestros cadetes es inferior al de los estudios hallados.

El porcentaje graso medio en hombres de deportes de equipo $^{10}$ (sin especificar la fórmula empleada, edad, ni categoría) es de $8,7-9,5$ y una suma de 6 pliegues de $63 \mathrm{~mm}$, siendo este sumatorio, similar a los resultados hallados $(66,96 \pm 16,60 \mathrm{~mm})$. En otro estudio ${ }^{24}$, también se puede observar que, para la modalidad deportiva del baloncesto, el sumatorio de 6 pliegues corresponde a $63,7 \mathrm{~mm}$, ligeramente superior al obtenido.

Otros porcentajes de interés son el muscular, el óseo y el residual, hallándose valores como $44,77 \%$, 18,24\% y $24,10 \%$ respectivamente en otros estudios ${ }^{18}$, que no varían demasiado de los observados $(41,75 \%, 17,46 \%$ y $28,11 \%$ respectivamente). Los deportistas profesionales tienen entre un $13-15 \%$ más de masa muscular que los no profesionales ${ }^{10}$ (sin especificar edad ni categoría). Teniendo en cuenta que el porcentaje muscular de alguien no entrenado se encuentra en valores aproximados al $37,5 \%{ }^{24}$, la diferencia con nuestros datos es más o menos notable.

Cabe destacar el índice músculo-óseo como indicador de crecimiento y aumento de masa muscular por kg óseo. El índice hallado $(<3,4)$ corresponde a un índice muy bajo, que indica poco desarrollo muscular ${ }^{22}$. Cabría la posibilidad de aumentar la masa muscular, aunque en esta categoría, todavía no han alcanzado su completo desarrollo osteomuscular, por lo que su estado físico y composición corporal irá cambiando y evolucionando ${ }^{12,25}$. 
Existe cierta escasez de estudios con datos relativos a los pliegues cutáneos así como sumatorios de pliegues, datos de composición corporal, etc. En varios de los artículos encontrados, únicamente se tiene en cuenta la talla y el peso 26,18 , dejando de lado datos tan importantes como el porcentaje de masa muscular, ósea y el somatotipo de cada jugador para un correcto rendimiento y desempeño del juego, como ocurre en otros estudios $2,10,27$.

Si analizamos la evolución del perfil del jugador de baloncesto en categorías superiores, destaca el cambio en los porcentajes de masa grasa y masa muscular. En un estudio realizado sobre jugadores indios interuniversitarios de 18 -

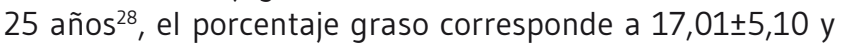
el porcentaje muscular a 82,99 $\pm 5,10$ (no se detallan porcentajes referentes al óseo y/o residual, por lo que el porcentaje muscular no es representativo), ambos muy superiores a nuestros datos.

En otro estudio que valora la composición corporal en jugadores de baloncesto en ligas LEB (05/06) y ACB (05/06, $06 / 07$ y $06 / 07)^{29}$, se encuentran valores de porcentaje de masa grasa de $9,9 \%, 11,9 \%, 8,8 \%$ y $10,4 \%$ respectivamente, todos ellos muy por debajo de nuestros datos sobre los cadetes. En cuanto a porcentaje de masa muscular se encuentra $49,2 \%, 47,7 \%, 47,9 \%$ y $49 \%$, respectivamente, valores por encima de los hallados en nuestro estudio.

La posición en el terreno de juego es otro factor a tener en cuenta en este deporte, ya que los movimientos y demandas fisiológicas en cada demarcación pueden modificar las capacidades de los jugadores ${ }^{8}$.

Encontramos referencias sobre la liga profesional $A C B^{8}$ rea-

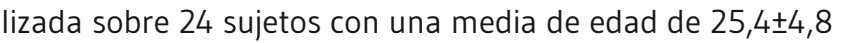
años, siendo los bases los de menor estatura y menor peso, aumentando ambos valores hasta llegar a los pívots, los cuales son más altos y también más pesados. En nuestro estudio, esta característica también se cumple, a diferencia del peso en el escolta y alero, siendo el peso del escolta superior. Esto puede ser debido a la poca muestra en el caso de la posición de escolta $(n=2)$.

En cuanto al porcentaje de masa grasa y muscular, en otro estudio ${ }^{9}$, las diferencias más notables se observan en el peso

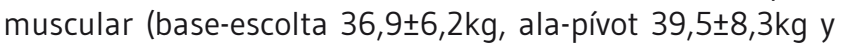
pívot $51,7 \pm 2,5 \mathrm{~kg}$ ), siendo muy superiores a las de nuestro estudio, sobre todo en los pívots. Otro estudio dedicado exclusivamente a los pívots de la liga $A C B^{30}$ recogiendo únicamente los datos de aquellos de raza blanca y españoles ( $n=10$ ) con edad de $24,73 \pm 1,55$ años, se observa que el porcentaje graso es de $12,12 \pm 2,05$, el muscular $47,03 \pm 1,82$ y el óseo $16,51 \pm 0,82$, siendo valores muy dispares a los obtenidos en nuestro estudio.
Otro punto importante es que el somatotipo nos acerca a conocer si el deportista posee el tipo corporal con éxito dentro de su modalidad deportiva y, si cabe, dentro de su posición de juego $0^{12,17}$. A la hora de caracterizar el somatotipo del jugador de baloncesto masculino cadete en su conjunto global, no hay un consenso. Algunos estudios ${ }^{18}$ lo establecen en mesomorfo balanceado con valores de endomorfia=3,14, mesomorfia $=3,99$ y ectomorfia=3,34. Otro estudio a una edad de 14 años $^{30}$, lo caracterizan como ectomesomorfo con valores de endomorfia=2,63, mesomorfia=3,39, ectomorfia 4,16 ; $y$, conforme van creciendo, pasan a mesoectomorfo $(15,16,17$ años) con valores de endomorfia=2,53 - 2,46 2,32; mesomorfia=3,4 - 3,49 - 3,65; y ectomorfia $=3,88-3,71$ - 3,49, respectivamente.

Con una media de edad de 15,35 $\pm 0,59$, el jugador de baloncesto cadete de nuestro estudio se caracteriza como ectomorfo balanceado (endomorfia=2,65; mesomorfia=2,58; ectomorfia=3,71). La descripción de este somatotipo se basaría en una predominancia de la ectomorfia, es decir, predomina la linealidad relativa moderada del jugador de baloncesto, acompañado de un bajo-moderado desarrollo músculo-esquelético relativo con pequeñas articulaciones y diámetros óseos y musculares relativos estrechos. A su vez, también se combina con una baja adiposidad relativa con poca grasa subcutánea ${ }^{17}$.

Analizando otros estudios sobre jugadores de baloncesto de categorías superiores, se observa que el somatotipo sigue sin ser un valor fijo, caracterizándose como mesomorfo balanceado ${ }^{18}$ con valores de 3,14; 3,99; 3,34 y como mesoectomorfo ${ }^{25,27}$ con valores de endomorfia $=2,59-2,51$; mesomorfia =3,92 - 4,54; y ectomorfia=3,08 - 3,06.

Además, se ha de valorar el somatotipo según posición de juego, pues en un estudio sobre jugadores de baloncesto masculino de élite internacional ${ }^{31}$, se clasifica al base como ectomesomorfo $(2,5 ; 5 ; 3)$, al escolta como ectomesomorfo $(2,1 ; 4,4 ; 3,5)$, al alero como ectomesomorfo $(2,2 ; 4,7 ; 3,3)$ y al pívot como mesoectomorfo $(2,8 ; 3,9 ; 3,7)$ (endomorfia; mesomorfia; ectomorfia). En cambio, en el estudio sobre pívots de la $A C B^{30}$, lo clasifica como ectomorfo balanceado $(2,67 ; 2,57 ; 4,03)$. Además, afirma que el somatotipo ideal del pívot es de 3,$02 ; 3,54 ; 3,17$.

Según nuestros datos, el cadete masculino del Valencia Basket Club se caracteriza con un base mesoectomorfo $(2,57 ; 3,38 ; 3,00)$, escolta ectomorfo balanceado $(2,38$; $2,49 ; 3,39)$, alero ectomorfo balanceado $(2,52 ; 2,40 ; 4,10)$ y pívot ectoendomorfo $(3,07 ; 2,31 ; 3,69)$. Comparando resultados, destaca la alta linealidad relativa de nuestros cadetes, observándose en las categorías superiores un mayor desarrollo muscular y, por tanto, mayor peso de la mesomorfia, y menor porcentaje graso. 
La obtención de un grado de desarrollo funcional y de cualidades físicas asociadas al baloncesto pueden ser utilizados para reflejar la evolución y/o dirigir el perfil óptimo del jugador de baloncesto. Los datos de este estudio pueden resultar de interés en la elaboración de programas específicos de entrenamiento e intervenciones a nivel dietético-nutricional, pudiendo tener efectos sobre el aumento o disminución de los valores de los pliegues cutáneos, peso y composición corporal. Podría ser interesante volver a valorar a los jugadores del Valencia Basket Club en el futuro y abarcar las pautas necesarias para conseguir dicho objetivo si no se alcanzara.

Las limitaciones del presente estudio son la insuficiencia de estudios encontrados en referencia a la categoría cadete, ya que la gran mayoría de ellos tratan con ligas profesionales y/o jugadores de élite internacional, que impide realizar una valoración representativa y completa.

\section{$\longrightarrow$ \\ CONCLUSIONES}

Las medidas de los cadetes masculinos del "Valencia Basket Club" no presentan grandes variaciones respecto a los datos para la misma categoría. La caracterización somatotípica global del jugador de baloncesto cadete es ectomorfo balanceado. Conocer el perfil cineantropométrico del jugador de baloncesto puede ser beneficioso para la elaboración de planes específicos de entrenamiento y nutrición, así como para reflejar la evolución y dirigirla hacia las características ligadas al rendimiento óptimo.

\section{Agradecimientos}

Agradecer, a nivel general, al Valencia Basket Club, el abrir sus puertas y participar en este estudio, así como a cada uno de los jugadores de baloncesto de la categoría cadete masculino partícipes. A nivel particular, agradecer al doctor Miguel Frasquet Pons su colaboración y atención, sin olvidar al preparador físico Pau Alcacer.

\section{conflicto de intereses}

Los autores expresan que no hay conflictos de intereses al redactar el manuscrito.

\section{BIBLIOGRAFÍA}

(1) FIBA. Official Basketball Rules | FIBA.COM [Internet]. FIBA. com. 2014 [citado 3 de abril de 2014]. Recuperado a partir de: http://www.fiba.com/pages/eng/fc/FIBA/ruleRegu/p/ openNodelDs/897/selNodelD/897/baskOffiRule.html

(2) NBA Media Ventures. Jugadores y Posiciones [Internet]. NBA. com. [citado 4 de abril de 2014]. Recuperado a partir de: http://www.nba.com/enebea/players.html

(3) Norton K, Olds T, editores. Anthropometrica: a textbook of body measurement for sports and health courses. 1a. ed. Sydney, Australia: UNSW Press; 1996.

(4) Esparza Ros F. Manual de cineantropometría. FEMEDE, editor. Madrid: FEMEDE; 1993.

(5) Petroski EL, editor. Antropometria: técnicas e padronizações. Porto Alegre, Brasil: Palotti; 2003.

(6) Mathews DK, Fox EL. Bases fisiologicas da educação fisica e dos desportos. 2a. ed. Rio de Janeiro, Brasil: Interamericana; 1979.

(7) Carter JEL. The Heath-Carter somatotype method. San Diego, California: San Diego State University; 1975.

(8) Ramos J, Rubio JA, Martínez F, Esteban P, Jiménez JF. Características fisiológicas, podológicas y somatométricas del jugador profesional de baloncesto. Arch Med Deporte. 2010; 27(136): 84-94.

(9) Cejuela Anta R, Pérez Turpin JA, Cortell Tormo JM, Chinchilla Mira JJ, Rivas Soriano J, Villa Vicente JG, et al. Correlations among anthropometric parameters, jump power, and position in professional basketball players. En Cáceres, España; 2007. Recuperado a partir de: http://rua.ua.es/dspace/ handle/10045/12278

(10) Urdampilleta A, Martínez-Sanz JM, Cejuela R. Indicadores del rendimiento deportivo: aspectos psicológicos, fisiológicos, bioquímicos y antropométricos. EFDeportes [Internet]. 2012 [citado 1 de mayo de 2014];17(173). Recuperado a partir de: http://www.efdeportes.com/efd173/indicadores-delrendimiento-deportivo.htm

(11) Stewart A, Marfell-Jones M. International standards for anthropometric assessment. ISAK, editor. Lower Hutt, New Zealand: International Society for the Advancement of Kinanthropometry; 2011.

(12) Cabañas MD, Esparza F. Compendio de cineantropometría. Madrid: CTO Editorial; 2009.

(13) Alvero J, Cabañas MD, Herrero de Lucas A, Martínez L, Moreno C, Porta J, et al. Protocolo de valoración de la composición corporal para el reconocimiento médico-deportivo. Documento de consenso del Grupo Español de Cineantropometría (GREC) de la Federación Española de Medicina del Deporte (FEMEDE). Versión 2010. Arch Med Deporte. 2010; 27(139): 330-46. 
(14) Withers RT, Craig NP, Bourdon PC, Norton KI. Relative body fat and anthropometric prediction of body density of male athletes. Eur ] Appl Physiol Occup Physiol. 1987; 56(2): 191200.

(15) Lee RC, Wang Z, Heo M, Ross R, Janssen I, Heymsfield SB. Totalbody skeletal muscle mass: development and cross-validation of anthropometric prediction models. Am J Clin Nutr. 2000; 72(3): 796-803.

(16) Rocha MSL. Peso ósseo do brasileiro de ambos os sexos de 17 a 25 años. Arq Anat Antropol. 1975; 1: 445-51.

(17) Carter L. Chapter 6 - Somatotyping. En: Anthropometrica: a textbook of body measurement for sports and health courses. 1a. ed. Sydney, Australia: UNSW Press; 1996. p. 147-70.

(18) García JG. Evaluación cineantropométrica de 101 jugadoresas cadetes de baloncesto. Estudio comparativo con una muestra aleatoria de alumnos-as de un Instituto de Formación Profesional. Arch Med Deporte. 1986; 3(11): 247-52.

(19) Stapff A. Chapter 14 - Protocols for the Physiological Assessment of Basketball Players. En: Physiological tests for elite athletes. Champaign, III, EE. UU.: Human Kinetics; 2000.

(20) Garrido-Chamorro RP, Sirvent-Belando JE, Gonzalez-Lorenzo M, Martin-Carratala ML, Roche E. Correlation between body mass index and body composition in elite athletes. J Sports Med Phys Fitness. 2009; 49(3): 278-84.

(21) Faulkner JA. Physiology of swimming and diving. En: Exercise physiology. New York: Academic Press; 1968. p. 415-43.

(22) Martínez-Sanz JM, Urdampilleta A. Protocolo de medición antropométrica en el deportista y ecuaciones de estimaciones de la masa corporal. EFDeportes [Internet]. 2012 [citado 1 de julio de 2014]; 17(174). Recuperado a partir de: http:// www.efdeportes.com/efd174/protocolo-de-medicionantropometrica-en-el-deportista.htm
(23) Strumbelj E, Erčulj F. Analysis of experts' quantitative assessment of adolescent basketball players and the role of anthropometric and physiological attributes. ] Hum Kinet. 2014; 42: 267-76.

(24) Martínez-Sanz JM. La valoración de la composición corporal. Curso ISAK nivel I; 2013.

(25) Aragoné M, Casajús JA. Modificaciones antropométricas debidas al entrenamiento. Estudios longitudinales. Arch Med Deporte. 1991; 8(32): 345-53.

(26) Vanderlei FM, Bastos FN, de Lemes IR, Vanderlei LCM, Júnior JN, Pastre CM. Sports injuries among adolescent basketball players according to position on the court. Int Arch Med. 2013; 6(1): 5

(27) Muñoz JA, Huici MJ, Marcos E. Estudio de la composición corporal y el somatotipo de deportistas sevillanos de alto rendimiento y comparación con la élite mundial. Arch Med Deporte. 1986; 3(11): 253-62.

(28) Koley S, Singh J. Anthropometric and physiological characteristic on indian inter-university basketball players. JPES. 2010; 28(3): 70-6.

(29) Tárrega L, Canda A. 5. Composición corporal del jugador de baloncesto. En: Bases científicas para la salud y un óptimo rendimiento en baloncesto. Madrid: Ergón; 2009.

(30) Galiano D, Ruiz C, Comaposada J. Estudio cineantropométrico en jugadores de baloncesto de raza blanca y negra. Apunts Med Esport. 1984; 21: 163-73.

(31) Martínez-Sanz JM, Urdampilleta A, Guerrero J, Barrios V. El somatotipo-morfología en los deportistas. ¿Cómo se calcula? ¿Cuáles son las referencias internacionales para comparar con nuestros deportistas? EFDeportes [Internet]. 2011 [citado 15 de junio de 2014];16(159). Recuperado a partir de: http:// www.efdeportes.com/efd159/el-somatotipo-morfologia-enlos-deportistas.htm 